\title{
Capítulo
}

6

\section{Realidade Aumentada no contexto de Computação Ubíqua: conceitos, características e ferramentas da plataforma Android}

\author{
Joel Machado Pires, João Soares de Oliveira Neto
}

\begin{abstract}
This chapter presents the main characteristics of Ubiquitous Computing and Augmented Reality and how these technologies can be used together to improve the user experience in urban space. Moreover, we discuss the features offered by the Android platform for the creation of Augmented Reality applications based on the location of users and objects placed in the environment where the users are. To illustrate the issues covered in this chapter, we present the process of designing, modeling and implementing the Intelligent Campus app - a set of resources and services based on Augmented Reality to provide information about buildings and other objects arranged on a university campus.
\end{abstract}

\section{Resumo}

Este capítulo apresenta as características principais da Computação Ubíqua e Realidade Aumentada e de que maneira estas tecnologias podem ser utilizadas em conjunto visando melhorar a experiência do usuário no espaço urbano. Além disso, são discutidos os recursos oferecidos pela plataforma Android para a criação de aplicativos de Realidade Aumentada baseados na localização dos usuários e de objetos presentes no ambiente. Para ilustrar os assuntos abordados neste capítulo, é detalhado o processo de concepção, modelagem e implementação do app Campus Inteligente, um conjunto de recursos e serviços baseados em Realidade Aumentada para oferecer informações sobre prédios $e$ outros objetos presentes num campus universitário.

\subsection{Introdução}

Ao prever um futuro em que a Computação estaria presente em todos os lugares, ambientes e contextos, além de estar disponível a todas as pessoas a todo momento, Weiser (1991) inspirou o surgimento de várias tecnologias e inovações necessárias para 
que este universo projetado se tornasse realidade. Conexões sem fio, soluções para comunicação móvel, processamento distribuído e interfaces naturais - baseadas em toque, voz e gestos - foram alguns dos avanços iniciais. Todavia, os anos seguintes testemunharam o surgimento de uma sucessão de novos conceitos e ferramentas concebidas para aumentar a sensação de onipresença.

Se algumas áreas/tecnologias fortaleceram a Computação Ubíqua e podem ser consideradas como habilitadoras - como é o caso Computação em Nuvem, Computação Vestível, GPS e sistemas de localização, sensores e tecnologias de identificação etc. outras áreas e tecnologias sofreram forte impacto, foram impulsionadas ou ganharam novos direcionamentos graças à Computação Ubíqua, como é o caso de Interação Humano-computador, Hardware, Arquitetura de Sistemas, Redes Sociais, Comércio Eletrônico, Realidade Virtual e também Realidade Aumentada (RA), entre outras.

A possibilidade de o indivíduo continuar conectado às redes de computadores mesmo em movimento, modificou na verdade a forma como interagimos com o ambiente e entre nós mesmos (REID et al., 2016). A miniaturização dos equipamentos permitiu o acesso a mais informação e a uma variedade de serviços dentro do perímetro urbano ou até mesmo na zona rural (REID et al., 2016): saúde, educação, monitoramento e segurança, localização e entretenimento, por exemplo. Nesse contexto, a RA pode ser vista como uma tecnologia urbana, ou seja, uma solução tecnológica concebida para melhorar a qualidade de vida urbana, em termos de energia, transporte, planejamento urbano, segurança, acesso a serviços, entre outros (JIANG et al., 2016).

RA é a combinação entre o mundo real e objetos virtuais, com os quais podem-se interagir em tempo real (DUBOIS; NIGAY, 2000; ZHOU; DUH; BILLINGHURST, 2008). Essa tecnologia tornou-se mais popular com a difusão de dispositivos móveis, cujas câmeras capturam imagens do mundo real e sobre as quais são inseridos objetos que adicionam informações à imagem real, ou seja, aumentam a realidade. As iniciativas de Cidades Inteligentes (CI) têm se beneficiado bastante do uso de RA. Tais iniciativas buscam melhorar a qualidade da governança de espaços urbanos e aumentar a qualidade de vida dos cidadãos a partir do oferecimento de tecnologias urbanas digitais (ALBINO; BERARDI; DANGELICO, 2015).

Em Cidade Inteligentes, a Realidade Aumentada pode ser usada para o reconhecimento de objetos no espaço urbano; inclusão de informações sobre prédios, praças, ruas etc.; mobilidade urbana, definição de rotas e navegação interna/externa; construção civil e arquitetura; poluição e meio ambiente; Tecnologia Assistiva e ferramentas de inclusão; manutenção e reparo; entretenimento; turismo; esporte e lazer; entre outros setores (KAJI et al., 2018; KAVAKLI, 2015; RASHID et al., 2017).

O objetivo deste capítulo é evidenciar e discutir os conceitos de RA e Computação Ubíqua, suas características e apresentar os recursos oferecidos pela plataforma Android para a criação de aplicativos de RA baseados na localização dos usuários e de objetos presentes no espaço urbano. Para ilustrar os assuntos abordados ao longo do minicurso, será detalhado todo o processo de concepção, modelagem e implementação do app Campus Inteligente, um conjunto de recursos e serviços baseados em RA para melhorar a experiência dos usuários de um campus universitário.

A Seção 6.2 aborda o tema Computação Ubíqua, enquanto que a Seção 6.3 apresenta os principais conceitos relacionados com Realidade Aumentada. Em seguida, 
na Seção 6.4, é discutido o estudo de caso: a criação de uma aplicação de Realidade Aumentada para um Campus Inteligente. A Seção 6.5 traz as considerações finais e aponta possibilidades de continuação de estudos nos temas abordados neste capítulo.

\subsection{Computação Ubíqua}

A Computação Ubíqua trata do desenvolvimento de soluções, na área de tecnologia da informação, que visam interagir com o usuário por meio de dispositivos que podem ser distribuídos no ambiente e que estão presentes no dia a dia das pessoas. Este paradigma computacional se contrapõe a ambientes restritos e controlados, como domicílios, escritórios e empresas, em que a conexão a redes de computadores se dá por meio de cabos ou conexões sem fio de curta distância. No contexto ubíquo, o usuário tem maior flexibilidade para se deslocar por longas distâncias, permanecendo o tempo todo conectado a redes de computadores (KUMAR, 2009).

Já que o indivíduo encontra-se constantemente imerso em ambientes que entrelaçam o real e o virtual e dada a onipresença computacional, a expectativa é que os dispositivos físicos e suas interfaces estejam diluídos, imperceptíveis e invisíveis de tão próximos que estão do cotidiano humano (WEISER, 1999). Embora a visão de Weiser tenha sido fortemente baseada em computadores, ele mesmo já sinalizava para o surgimento de dispositivos vestíveis - ou wearable devices, em inglês. Tais dispositivos ganharam grande notoriedade a partir do lançamento de vários modelos e aplicações, que foram amplamente adotados para fins gerais e específicos, tais como monitoramento de saúde, entretenimento, segurança, tecnologia assistiva, identificação etc.

Garantir o grau de transparência sugerido para sistemas ubíquos - para a comunicação e mobilidade, por exemplo - exige um enorme investimento no desenvolvimento de sistemas distribuídos, interface de usuários e projeto de interação. As características que diferenciam sistemas ubíquos de outros sistemas é que eles são executados em ambientes personalizáveis, centrados no usuário e cuja interação acontece de maneira menos perceptível. Além disso, são sistemas sensíveis ao ambiente, ou ao contexto em que estão inseridos. Quanto mais conscientes do contexto ao seu redor, mais estes sistemas são capazes de se adaptarem, atuarem e controlarem o ambiente. De maneira resumida, os requisitos de sistemas ubíquos são (POSLAD, 2009; WEISER, 1999):

- Os dispositivos precisam estar conectados à rede, distribuídos e acessíveis de maneira transparente;

- A interação humano-computador precisa estar o mais implícita, oculta e camuflada possível;

- Necessitam ser sensíveis ao contexto, a fim de otimizar o seu funcionamento no ambiente em que se encontra;

- Os dispositivos podem funcionar de maneira autônoma, sem interferência humana e auto-gerenciáveis;

- Podem lidar com uma multiplicidade de ações e interações dinâmicas. Isso pressupõe alguma forma de Inteligência Artificial a fim de tratar:

○ interações incompletas e não-determinísticas;

- cooperação e competição entre membros da mesma equipe; 
○ interações sofisticadas a partir do compartilhamento de contexto, semântica e metas.

Poslad (2009) sintetiza que os elementos de um sistema ubíquo podem ser agrupados em dois clusters: (a) contexto pessoal, econômico e social centrado no ser humano; e, (b) uma camada física formada por um ecossistema de elementos vivos (seres humanos) e inanimados (dispositivos e hardware). Para o autor, tais elementos não são mutuamente exclusivos; pelo contrário: eles se sobrepõem e precisam ser combinados.

Muitas das atividades humanas podem ser automatizadas por máquinas em sistemas ubíquos, permitindo que informações e serviços sejam acessados quando e onde necessário. Dadas as suas características intrínsecas e a fim de atender aos seus requisitos, os sistemas ubíquos devem lidar com algumas limitações (ALENCAR et al., 2014; KUMAR, 2009; ROLIM et al., 2015):

- Instabilidade, perda de conexão ou área de sobra;

- Confiabilidade e disponibilidade dos recursos computacionais;

- Restrições de interação, como tela pequena ou falta de botões em dispositivos vestíveis;

- Limitações de hardware, como processamento, memória e espaço para armazenamento reduzidos;

- Autonomia de energia e bateria;

- Heterogeneidade de dispositivos e sistemas (fornecedores e plataformas diferentes);

- Segurança e privacidade em ambientes distribuídos;

- Padronização na comunicação entre dispositivos de fornecedores diferentes;

- Gerenciamento e coordenação de atividades entre dispositivos diversos

O desenvolvimento de aplicações ubíquas, atendendo às especificidades destas aplicações, requer plataformas e ferramentas específicas, assim como a infraestrutura em que essas aplicações são executadas. Empresas do setor de software e de hardware já fornecem ambientes robustos para que desenvolvedores criem suas soluções e para que tais soluções sejam executadas oferecendo aos usuários as suas funcionalidades. A plataforma Android, por exemplo, foi projetada de maneira a oferecer (a) recursos para desenvolvedores e (b) um ambiente para execução de aplicações ubíquas garantindo interoperabilidade, mobilidade, heterogeneidade, adaptabilidade, interação implícita, auto-gerenciamento e recursos de Realidade Aumentada (HASSAN, 2008).

Em particular, os smartphones Android fazem parte do cotidiano da maioria dos usuários de dispositivos móveis (NABILA POPAL; RYAN REITH, 2021). A interação com estes dispositivos é por meio de tela sensível ao toque e, apesar de possuírem telas majoritariamente pequenas, têm poder de processamento suficiente para processamento gráfico, isso possibilita inúmeras aplicações de entretenimento. Ainda, muitos deles possuem GPU e compatibilidade com a biblioteca gráfica Vulcan, que torna a interação do usuário mais fluida, visto as vantagens desses recursos para o aumento de performance.

O desenvolvimento para Android pode ser feito com diversas linguagens de programação, como Python, Kotlin, Java, C\#, C/C++. Java e JavaScript com React 
Native. As mais usuais são Java e Kotlin, utilizando ambiente de desenvolvimento Android Studio, que formam uma poderosa ferramenta. Aplicações ubíquas voltadas para diversos setores foram desenvolvidas na plataforma Android, tais como: saúde (GEMAN et al., 2018; HII; CHUNG, 2011), automação residencial (PIYARE, 2013), educação (SHANMUGAPRIYA, 2011), entre outros setores.

\subsection{Realidade Aumentada}

Quando um usuário é inserido em um ambiente virtual, diz-se que a tecnologia utilizada é Realidade Virtual (RV), quando o ambiente recebe objetos virtuais, diz-se que a tecnologia utilizada é Realidade Aumentada. Um exemplo de aplicação de RV é os óculos VR, ele permite que o usuário visualize um ambiente virtual e que se pareça que esteja dentro desse ambiente. Os filtros que inserem elementos nas fotos, figurinhas, máscaras, perucas, óculos, etc., presentes no aplicativo Instagram (INSTAGRAM, [s.d.]), é um exemplo de aplicação de RA.

Sabe-se que a tecnologia tem avançado na área digital e as que envolvem RA estarão cada vez mais presentes no cotidiano. No entretenimento, os jogos eletrônicos, filmes e aplicativos de redes sociais estão contanto com esta tecnologia ("Pokémon GO", 2016; RAUSCHNABEL; ROSSMANN; TOM DIECK, 2017). Também, RA tem potencial em outros contextos, como na educação e em aplicações em Cidades Inteligentes (MADI; ALBAKRY; IBRAHIM, 2020).

No entanto, o desenvolvimento de aplicações de RA tem suas barreiras computacionais, seja por limitações de hardware, seja pela complexidade de algumas tarefas, e a percepção do ambiente pelo dispositivo é uma delas.

Quando um usuário está interagindo com um ambiente, por meio de uma aplicação mobile, as posições dos objetos no ambiente mudam em relação ao dispositivo, de acordo com o movimento dado a ele pelo o usuário, por exemplo, quando o usuário gira o celular, balança ou aponta para algum lugar. Essa dinâmica deve ser considerada nas aplicações, podendo ser feita com a exploração dos recursos disponíveis nos dispositivos (GRUBERT; GRASSET, 2013).

Com auxílio dos sensores, é possível aumentar o dinamismo e realismo nas aplicações. A câmera pode fornecer imagens em tempo real; o GPS fornece a localização instantânea do dispositivo; o sensor magnético junto com o acelerômetro e giroscópio orientam o dispositivo no ambiente; enquanto a tela sensível ao toque recebe comandos do usuário e retorna as imagens da aplicação.

Com isso, uma forma de orientar o dispositivo no ambiente, fazendo com que a interação seja dinâmica, é a combinação dos sensores magnético, giroscópio e acelerômetro, como sugerido por Lawitzki (2011) em seu tutorial na Web. Visão computacional é outra forma, que se tornou possível com o avanço do hardware dos dispositivos móveis.

Por outro lado, outro requisito das aplicações de RA, no caso das aplicações mobile, são as alterações nas imagens obtidas pela câmera. Essas alterações podem ser desenhos de objetos 2D e 3D, por exemplo. Por sua vez, estes desenhos demandam processamento gráfico e podem ser feitos com bibliotecas gráficas, como OpenGL ES e Vulkan. 
Estas ferramentas podem ser utilizadas nos smartphones Android. Ademais, a plataforma Android é a mais frequente e possui a maior variedade de hardware, mas tendo câmeras, GPS, tela sensível ao toque, sensor magnético, acelerômetro, giroscópio, acesso à internet e processamento de alta performance, como características em comum (KO; CHANG; JI, 2013). Apesar disso, muitas vezes, ainda não é possível utilizar orientação com sensores, visão computacional, e processamento gráfico ao mesmo tempo. Então, uma comparação das vantagens da utilização de um ou outro recurso pode ser feita, para que a aplicação funcione.

Existem ferramentas, de nível mais alto, para desenvolvimento de aplicações de RA na plataforma Android, considerando as limitações de hardware, como as "Tool Kits" para $\mathrm{RA}^{1}$, de forma similar, têm-se o Vuforia $\mathrm{SDK}^{2}$. Estas podem ser utilizadas como soluções pré-modeladas para aplicações de RA. A vantagem é que boa parte das integrações com sensores, visão computacional e processamento gráfico já estão desenvolvidas e otimizadas, assim, o desenvolvedor da aplicação de RA em si não precisa se preocupar muito com aspectos da computação gráfica e otimização dos algoritmos. Isso permite um desenvolvimento mais rápido e suscetível a menos erros.

Portanto, vê-se que as aplicações de RA estão tendendo para dispositivos móveis, consequentemente, para a plataforma Android, por ser mais popular. Além disso, as limitações ainda existentes estão sendo minimizadas por meio de melhoramento de hardware e soluções de software. Com isso, aplicações de RA estão cada vez mais comuns.

\subsubsection{Aplicações e exemplos}

O Livro Mágico é um tipo de aplicação de RA, usando-se de um livro para inserir elementos estáticos, que podem variar com a movimentação das páginas. De forma análoga, projetores podem inserir imagens de objetos, estáticos ou animados, em uma superfície real, como mostram as figuras Figura 6.1 (a) e Figura 6.1 (b) (CRAIG, 2013).

\footnotetext{
${ }^{1}$ ARToolKit; ARToolKit for Android

${ }^{2}$ Vuforia SDK
} 


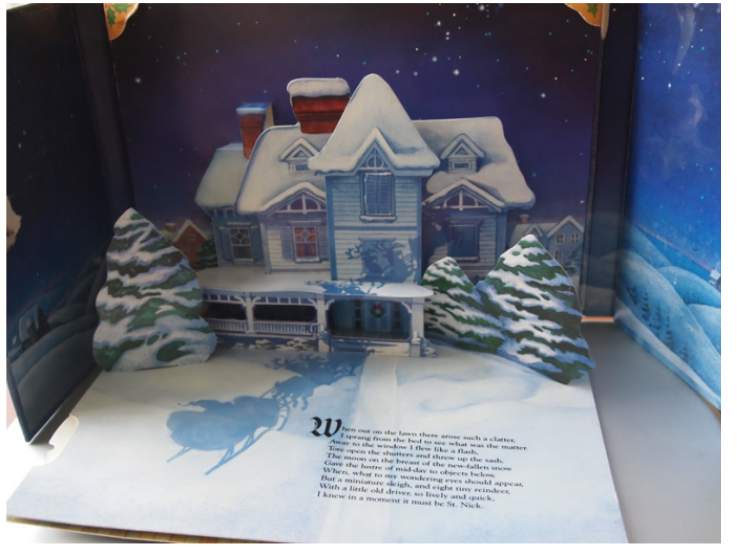

(a)

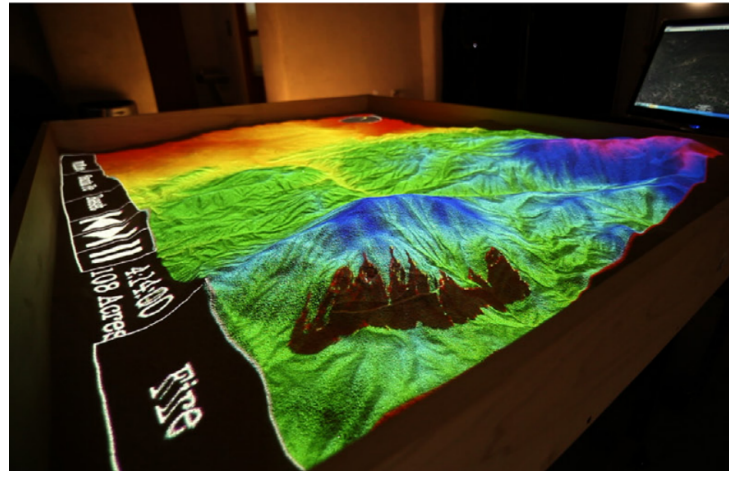

(b)

Figura 6.1. Exemplo de uma aplicação de RA a estilo de Livro Mágico. Fonte: (CRAIG, 2013)

Quando se usa um espelho para refletir uma imagem real em conjunto com elementos adicionais, tem-se o Espelho Mágico, a exemplo, uma pessoa pode se olhar no espelho, projetando uma peruca que foi desenhada no próprio espelho físico. Assim como o Livro Mágico, este estilo tem suas similaridades com aplicações mais atuais, como a projeção de uma barba, peruca ou qualquer um objeto, em uma imagem tirada com a câmera, utilizando o aplicativo Instagram, por exemplo. A Figura 6.2 mostra um exemplo desse estilo.

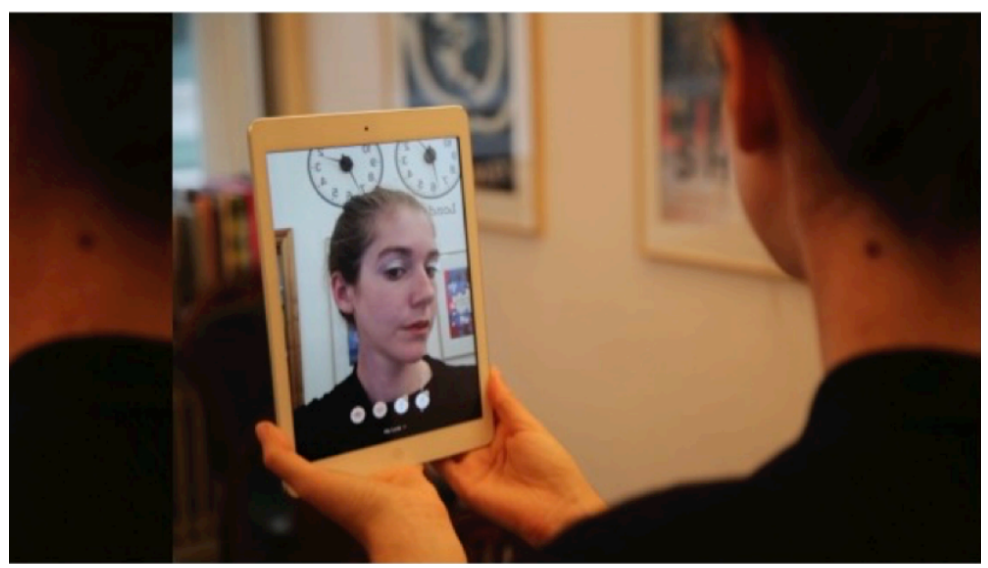

Figura 6.2. Aplicação em dispositivo móvel que insere maquiagem na imagem obtida pela câmera frontal. Fonte: (JAVORNIK et al., 2016)

Um dos estilos mais frequentes é o Janela e Portas Mágicas, no geral, apresentamse imagens animadas em uma janela no ambiente e esta janela pode ser um cartaz com alguma imagem que servirá de espaço para conter alguma animação. O Lente Mágica é um estilo que abrange a maioria das aplicações de RA, pois envolve o uso de telas, que 
podem ser de computadores e smartphones. A Figura 6.3 mostra um exemplo que combina o estilo Janelas e Portas Mágicas com o Lente Mágica, feito por KAN, TENG e CHOU (KAN; TENG; CHOU, 2009), utilizando QR code como marcador.

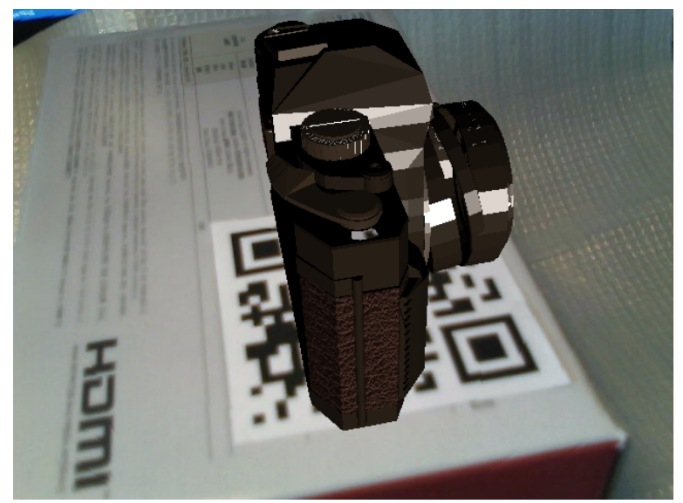

Figura 6.3. Tela de uma aplicação que utiliza uma imagem com QR Code como janela para inserir um objeto virtual na imagem, em tempo de execução. Fonte: (KAN; TENG; CHOU, 2009)

Entendendo estes estilos, pode-se estendê-los e combiná-los para aplicar RA nos diversos contextos. Como MADI et al, que constataram que um aplicativo de RA estimula a criatividade de crianças no aprendizado de Hajj, através de um estudo de caso de uma aplicação de RA nesse contexto. Esta aplicação foi em dispositivos móveis, para o auxílio no aprendizado de Hajj de crianças na Malásia, baseada no construtivismo.

IRWANSYAH et al. (IRWANSYAH et al., 2018) fizeram um aplicativo para estudo de estrutura molecular, na disciplina de Química, com uso de RA ao estilo Janelas e Portas Mágicas. Mostraram uma metodologia com uso de Sketchup, para modelar objetos 3D; Corel Draw X5, para criar o marcador; e, Unity para construir um jogo executável no sistema Android.

RUAN e JEONG (RUAN; JEONG, 2012) mostraram o uso de $Q R$ code, como marcador, para aplicações de RA em smartphones Android. Discutiram as vantagens comparado com o ARToolKit, onde colocou-se evidencia a possibilidade de guardar e ler informações, por meio de codificação e decodificação, com processamento mais rápido. A aplicação que eles mostraram foi feita com uso da câmera de smartphone para capturar imagens de $Q R$ code no ambiente. A parte de identificação do código, extração de informações e inserção de um objeto 3D na imagem, foram desenvolvidas com auxílio das bibliotecas OpenCV e OpenGL.

RODELLO e BREGA (RODELLO; BREGA, 2011) fizeram uma revisão sistemática sobre realidade aumentada no contexto de marketing. Entre os trabalhos discutidos, mostraram um aplicativo mobile de mapeamento. $\mathrm{O}$ funcionamento desta aplicação consiste em mostrar ícones com nomes dos locais, junto com as distâncias em tempo, como mostra a Figura 6.4. Pinto e Centeno (2012) também implementaram uma aplicação similar à da aplicação do Bradesco em dispositivos móveis. 


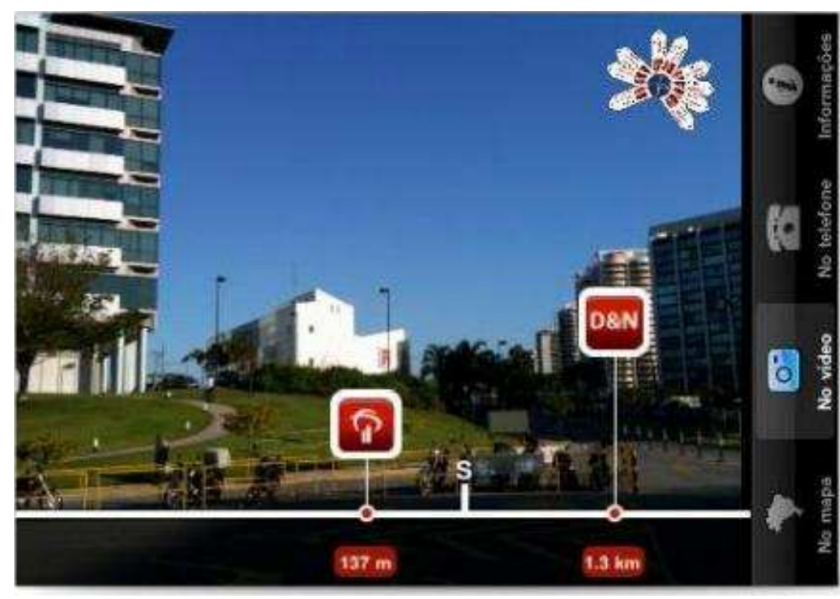

Figura 6.4. Tela da aplicação de mapeamento do Bradesco implementada na plataforma mobile iOS. Fonte: (RODELLO; BREGA, 2011)

BADOUCH et al. (BADOUCH et al., 2018) fizeram uma revisão bibliográfica sobre trabalhos de RA, no contexto de cidades inteligentes, onde é discutido as limitações e dificuldades a serem vencidas, que aparecem nos trabalhos revisados. Entre eles, destaca-se um aplicativo feito com a ferramenta ARKit, para smartphones $i O S$, que insere um objeto 3D em tempo de execução em uma imagem obtida pela câmera. Um segundo exemplo de aplicação, é um sistema que recebe dados de diversos sensores distribuídos na cidade de Santander (Cantábria, Espanha) e envia esses dados para um dispositivo móvel em tempo real, para que o usuário seja informado sobre o tempo, pontos de ônibus, serviços de motoqueiros, etc. Enquanto o celular captura imagens da rua, essas informações são transmitidas na tela, inseridas nas imagens. O terceiro, é um óculos inteligente, Google Glass, criado pela Google, que usa câmera, microfone e acesso a internet para dispor objetos no ambiente de forma virtual, para o usuário. Uma das dificuldades destacadas é em relação ao poder de processamento do dispositivo para executar os aplicativos de RA, onde o público pode não ter dispositivos na faixa ideal de hardware. A privacidade e segurança também é um fator que pode ser problema para alguns protótipos, como o Google Glass.

KOUNAVIS et al. (2012) Examinaram o estado da arte na área de RA, para dispositivos móveis, avaliaram os objetivos, limitações tecnológicas de vários trabalhos e propuseram um modelo de desenvolvimento para aplicações nessa área, para o turismo. Eles citaram várias ferramentas com potencial para desenvolver aplicações com realidade aumentada, entra-se em destaque a IN2AR, que faz uso de características de imagens em geral, onde elas podem ser usadas como marcador ao invés de somente $Q R$ code, porém, não está disponível para smartphones Android. Entre as aplicações destacadas no contexto de turismo, tem-se o Tuscany+(iOS) que obtém as informações dos locais que são visitados na internet e desenha elas na tela, que é semelhante a ideia proposta do presente trabalho. Outro exemplo é o Basel AR Tourist Guide, que dispõe de informações disponíveis de museus, restaurantes, hotéis e shopping centers, da cidade de Basel na tela. Já o StreetMuseum, coloca imagens históricas das ruas na tela quando o usuário aponta a câmera para elas. 
Cabe ressaltar ainda, que com o avanço da tecnologia de hardware, aplicações de RA com uso de Deep Learning tornaram-se possíveis, tanto para computadores de mesa, quanto em dispositivos móveis. WIBOWO, HARTANTO e WARTANTO (2020) desenvolveram uma aplicação para detecção de câncer de pele, na plataforma Android, que é uma aplicação de RA ao estilo Lente Mágica, como ilustrado na Figura 6.5, o resultado é um retângulo desenhado na tela, com um nome e probabilidade de acerto.

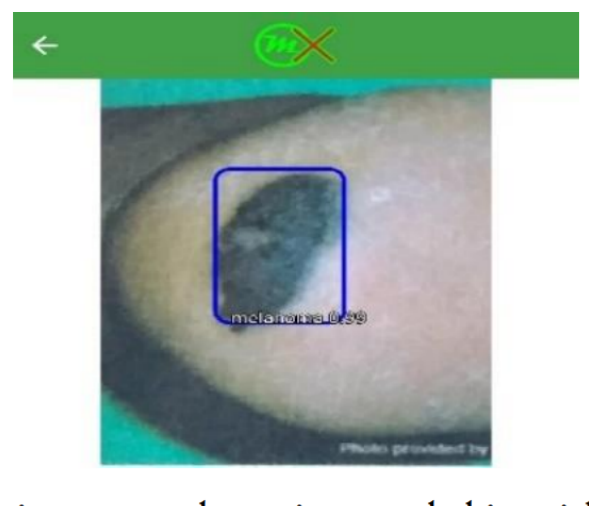

Figura 6.5. Aplicação Android que detecta câncer de pele, através de uma imagem. Fonte: (WIBOWO; HARTANTO; WIRAWAN, 2020).

Também, utilizando a técnica de Deep Learning, CHEN et al e JEONG (CHEN et al., 2021; JEONG, 2020), cada, fizeram aplicações que detectam um objeto na imagem real e desenham alguma informação. Dessa forma, pode-se estender o uso de marcadores nas imagens, para qualquer objeto real, como demonstrado pelos autores.

Então, percebe-se uma relação entre os estilos de RA, destacados por CRAIG (2013), com os trabalhos da literatura. Além disso, nota-se a diversidade de aplicações de RA, assim como o leque de ferramentas existentes.

\subsection{Exemplo prático: Campus Inteligente}

Uma consequência dos projetos de Cidades Inteligentes foi o surgimento dos Campus Inteligentes: regiões universitárias formadas por prédios e instalações voltadas para atividades acadêmicas de ensino, pesquisa e extensão voltadas para a graduação e para a pós-graduação e equipadas com uma variedade de serviços digitais ubíquos e a disponibilidade de informações contextuais para a comunidade de usuários deste espaço. A bem da verdade, conforme defende Ferreira e Araújo (2018), o Campus Inteligente pode ser comparado a Cidades Inteligentes menores, que buscam atender às demandas dos seus residentes oferecendo serviços de forma eficiente, reduzindo custos, mas, num ambiente controlado. Nesse tipo de projeto, não se pode perder de vista o contexto educacional em que está inserido e a necessidade de proporcionar experiências de qualidade no que diz respeito ao ensino-aprendizagem, mas, também em relação aos serviços que orbitam em torno das questões acadêmicas: biblioteca, restaurante, mobilidade etc. 
No contexto de Campus Inteligentes, várias universidades têm oferecidos uma gama de aplicações e funcionalidades - geralmente sob a forma de aplicativos para smartphones com diversas finalidades (FERREIRA; ARAÚJO; SANTOS, 2018; TAROUCO et al., 2017): conscientização ambiental, controle de tráfego, ensino, saúde, segurança, infraestrutura, por exemplo.

Pensando no contexto prático de turismo e mobilidade, verificou-se que existem poucas soluções que permitem, ao usuário, o aumento da percepção de um ambiente externo desconhecido. No entanto, de forma similar aos trabalhos citados, pode-se aplicar RA para tal objetivo.

Nesse contexto, limitando-se ao caso do campus de Cruz das Almas - Bahia, da UFRB, pensou-se numa aplicação Android que mostra informações a respeito dos prédios. Tais informações são horário de abertura e fechamento, e o tipo de serviços que o oferecidos nos prédios.

Esta aplicação deve ser na plataforma Android, para abranger um maior número de usuários e, ainda, proporcionar boa experiência aos usuários. Para isso, necessita-se de uma interface limpa e um algoritmo de média ou baixa demanda de processamento, para evitar travamentos durante a execução.

Com relação à modelagem do sistema, considere a Figura 6.6, que mostra a disposição dos recursos utilizados. A ideia é fazer uma aplicação Android que obtém imagens da câmera e informações de acelerômetro, sensor geomagnético e GPS, em tempo de execução, daí, exibir uma imagem do ambiente real com um objeto virtual, que indiquem informações a respeito do local de onde o usuário está.

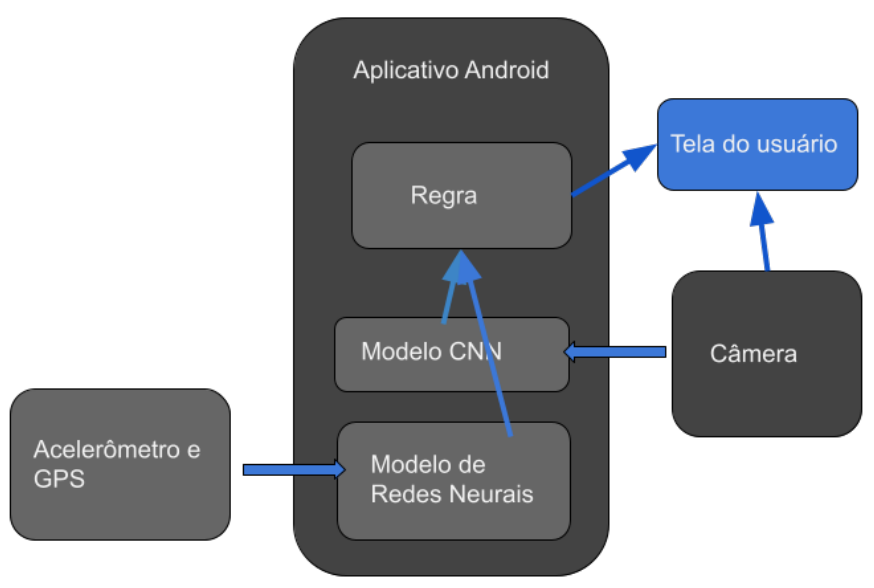

Figura 6.6. Disposição dos componentes no sistema de RA, na plataforma Android. Fonte: Autoria própria. 
Naa Figura 6.6, o componente "Modelo CNN" é um modelo de Redes Neurais Convolucionais para detecção de objetos, com a arquitetura Mobile Net V2 (YOUNIS et al., 2020). Esta arquitetura permite obter as coordenadas da posição de um objeto em uma imagem, com estas coordenadas, é possível desenhar um objeto virtual, ao estilo Janela e Portas Mágicas, por exemplo.

Para treinar um modelo de detecção de objetos, deve-se construir uma base de dados para ajuste e testes. No presente trabalho, utilizou-se 320 imagens, distribuídas entre 9 tipos diferentes de blocos do campus de Cruz das Almas, da UFRB, representadas pela Figura 6.7. Cada imagem foi anotada com o programa LabelImg (TZUTALIN, 2015), com retângulos que contenham os blocos.

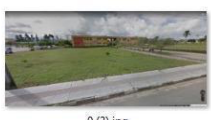

o(2).jpg

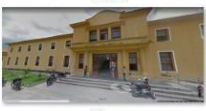

$0 . j p g$

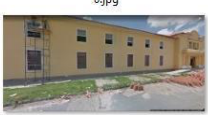

1(4).jpg

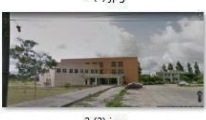

2(2).jpg

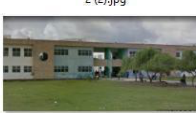

2(5).jpg

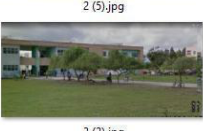

3(2).jpg
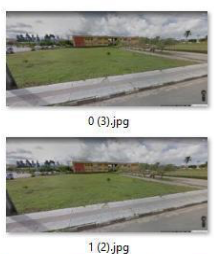

(2).jpg
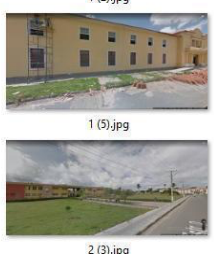

2(3).jpg
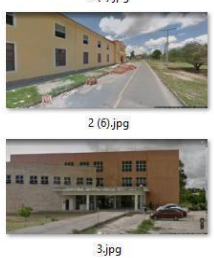

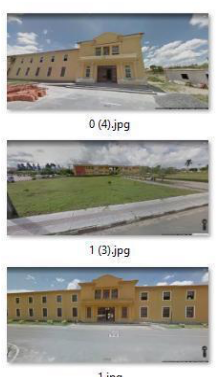

1.jpg

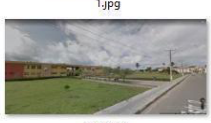

2(4).jpg

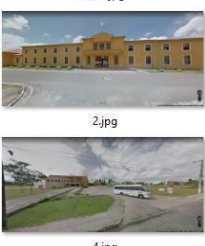

Figura 6.7. Parcela do conjunto de imagens montado para treinamento e teste do modelo de detecção de objetos. Fonte: Google Maps (GOOGLE MAPS, 2021)

Um passo a passo, em forma de tutorial, para treinamento de um modelo de detecção de objetos utilizando a arquitetura Mobile Net v2 pode ser encontrado no repositório do TensorFlow.

O componente "Modelo de Redes Neurais" é um regressor, que tem os valores instantâneos do magnetômetro e acelerômetro como entrada, e retorna o menor ângulo que à tela do celular faz com o vetor sul->norte geométrico $(\mathrm{N}-\mathrm{S})$. Este ângulo serve para confirmar o bloco identificado pelo modelo de detecção de objetos, através de uma regra. Este modelo de regressão é necessário, pois os valores lidos pelo magnetômetro também variam de acordo com a inclinação do eixo $y$ do celular em relação ao normal do globo terrestre, mesmo mantendo a direção da normal à tela $\left(\widehat{n_{C}}\right)$ constante, em relação ao vetor $N-S$, como mostrado na Figura 6.8. Dessa forma, outros sensores devem auxiliar na 
definição da direção de $\widehat{n_{C}}$ em relação às coordenadas geométricas, como o sensor acelerômetro, que retorna as intensidades da gravidade nos três eixos do celular.

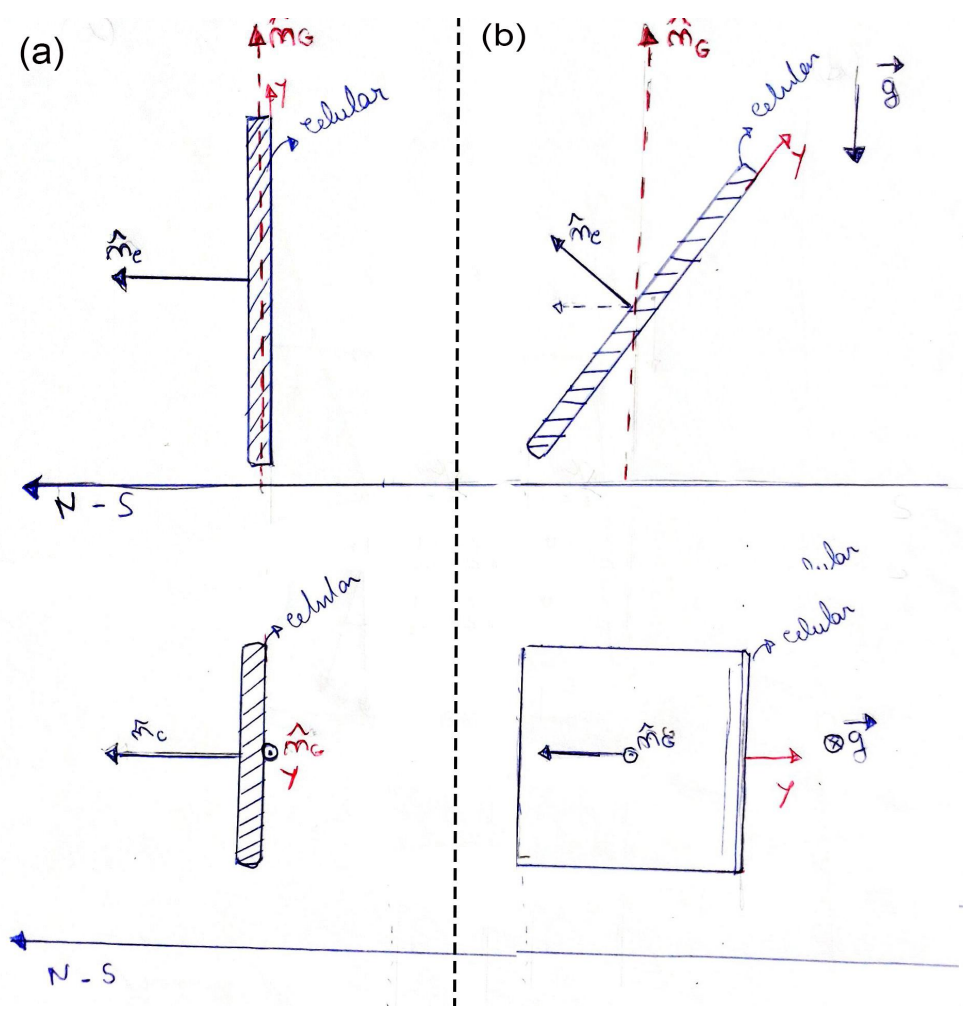

Figura 6.8. Diagrama de vetores estudados no smartphone, com relação ao ambiente e as leituras dos sensores. $\hat{n}_{G}$ é o vetor unitário normal à superfície terrestre; $\widehat{n_{C}}$ é o vetor unitário normal à superfície paralela à tela do celular. $g$ é o vetor aceleração gravitacional. (a) Celular posicionado de forma vertical. (b) Celular inclinado.

O sensor magnético também retorna intensidades do campo magnético terrestre para os três eixos do dispositivo. Então, quer-se obter um conjunto de parâmetros que definem a relação entre essas 6 variáveis dos sensores, para definir uma angulação de $\widehat{n_{C}}$ com o vetor $N-S$, no presente problema, um modelo de redes neurais com duas camadas totalmente conectadas é suficiente. Este modelo é demonstrado pela Figura 6.9. O componente "Regra" tem o papel de receber os resultados da detecção dos blocos nas imagens obtidas pela câmera do celular, confirmar, por meio do resultado do modelo de regressão e decidir se desenha ou não na tela. Este desenho é feito com a biblioteca Canvas, do Android. 


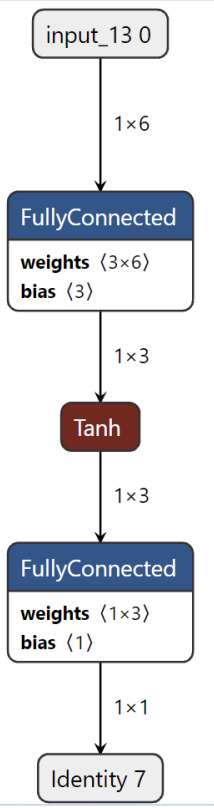

Figura 6.9. Arquitetura do modelo de regressão. Fonte: autoria própria.

A Figura 6.10 ilustra como a regra principal funciona. Este processo se repete continuamente, enquanto o usuário estiver na tela de execução. 


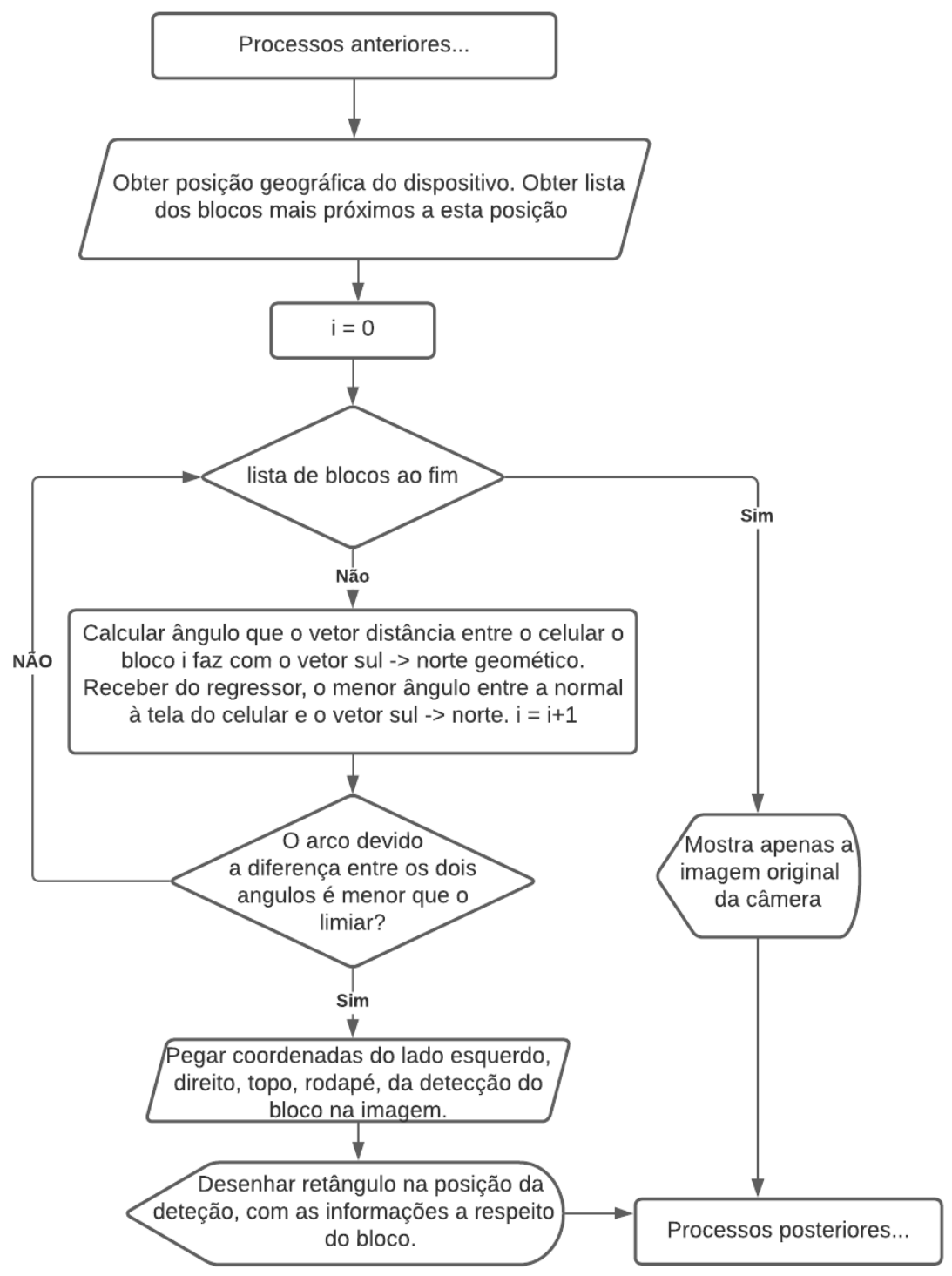

Figura 6.10. Fluxograma principal do aplicativo Android. Fonte: Autoria própria.

Após modelagem do sistema, segue-se para a implementação. Para isso, utilizase o ambiente de desenvolvimento Android Studio, com as linguagens Java e Kotlin como principais. Com relação desenvolvimento e treinamento dos modelos de Machine Learning, para detecção e regressão, foi utilizada a linguagem de programação Python.

A interface principal da aplicação implementada é mostrada pela Figura 6.11. A implementação teve como base a aplicação de exemplo do TensorFlow Lite, para detecção de objetos, disponível no repositório (TENSORFLOW COMMUNITY, [s.d.]). 


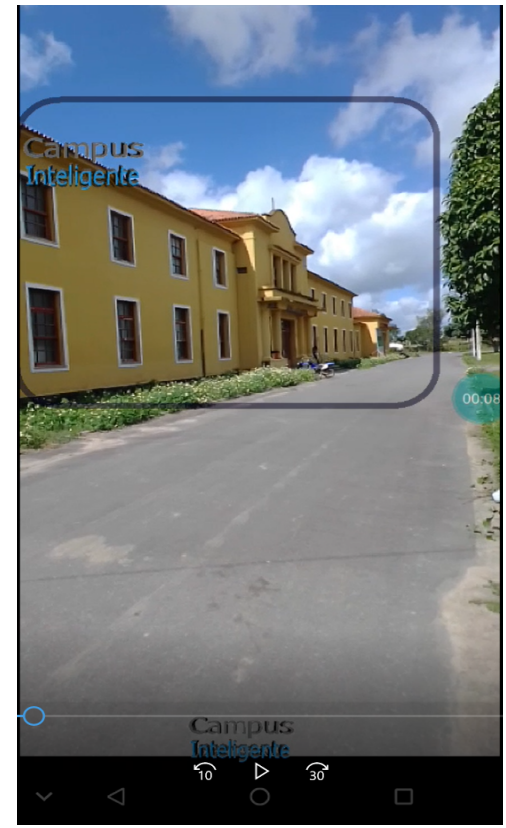

Figura 6.11. Frame de vídeo de captura de tela durante teste em campo da aplicação, com regressor desativado. Fonte: autoria própria.

As figuras Figura 6.12 (a) e Figura 6.12 (b) mostram os resultados da aplicação Android implementada.

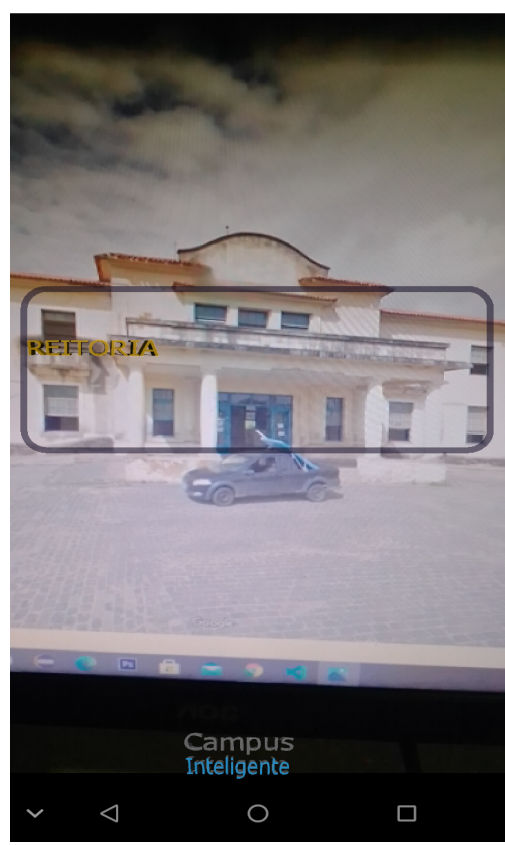

(a)

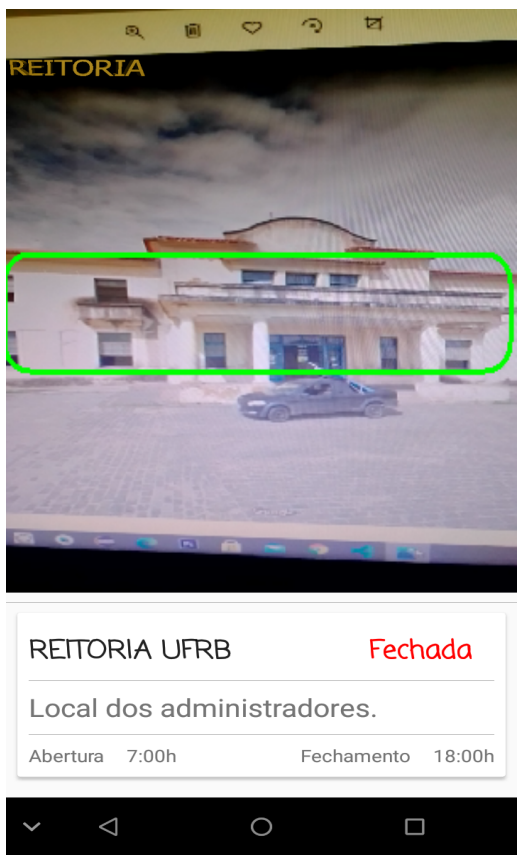

(b)

Figura 6.12. (a) Tela principal do aplicativo ao detectar um prédio. (b) Tela de "mais informações". Fonte: autoria própria. 


\title{
6.5. Considerações finais
}

Baseada na informação, comunicação e no conhecimento, a sociedade contemporânea é caracterizada pela conexão permanente às redes de comunicação. A grande maioria das atividades cotidianas realizadas pelos seres humanos nos dias de hoje são executadas online. Isso se dá graças a uma infraestrutura de hardware, software e telecomunicações conhecida como Computação Ubíqua. Conectados a smartphones, laptops, wearables podemos acessar a serviços e aplicações, como Realidade Aumentada, a qualquer hora em qualquer lugar. A Computação Ubíqua proporciona tecnologias baseadas em dispositivos, conexão a redes e software que permitem aos usuários, mesmo em movimento e longe de cabos de conexão, acessarem aplicações e serviços armazenados remotamente.

Por outro lado, a Realidade Aumentada possibilita ampliar os mecanismos de visualização da informação, bem como enriquecer o conteúdo apresentado ao usuário com o acréscimo de mais dados que a princípio não estão visíveis no ambiente real. Setores como educação, entretenimento e saúde têm disponibilizado soluções inovadoras baseadas em RA tornando os processos de ensino-aprendizagem, formação, diversão e compreensão do funcionamento do próprio corpo mais fáceis, agradáveis e divertidos.

Podemos acessar aplicações de Realidade Aumentada nos deslocando e recebendo informações na tela sobre prédios, ruas, avenidas e praças. A associação de RA e Computação Ubíqua tem mostrado grande potencial sobretudo no desenvolvimento de aplicações baseadas na localização do usuário no espaço urbano e em soluções para Cidades Inteligentes. Jogos como Pokemon Go, soluções para turismo e instrumentos para visualização da informação são alguns exemplos de aplicação de RA no contexto de Computação Ubíqua.

Neste capítulo foi mostrado os conceitos de Realidade Aumentada e Computação Ubíqua. Com isso, fez-se uma revisão de literatura, avaliação de ferramentas disponíveis e possíveis aplicações na plataforma Android, assim como seu potencial para a área. Mostrou-se também, o processo de planejamento, modelagem e implementação do aplicativo Campus Inteligente, utilizando ferramentas recentes, como Deep Learning $e$ a linguagem de programação Kotlin.

Como próximo passos no percurso do aprendizado convergindo os assuntos Computação Ubíqua e Realidade Aumentada sugere-se incluir estudo de Internet das Coisas e sensores.

\section{Referências}

ALBINO, V.; BERARDI, U.; DANGELICO, R. M. Smart Cities: Definitions, Dimensions, Performance, and Initiatives. Journal of Urban Technology, v. 22, n. 1, p. 3-21, 2 jan. 2015.

\author{
ALENCAR, T. S. DE et al. Addressing the Users' Diversity in Ubiquitous \\ Environments through a Low Cost Architecture. SpringerLink. Anais... In: \\ INTERNATIONAL CONFERENCE ON UNIVERSAL ACCESS IN HUMAN- \\ COMPUTER INTERACTION. Springer, Cham, 22 jun. 2014. Disponível em:
}


<https://link-springer-com.ez67.periodicos.capes.gov.br/chapter/10.1007/978-3-31907446-7_43>. Acesso em: 22 abr. 2017

BADOUCH, A. et al. Augmented Reality services implemented within Smart Cities, based on an Internet of Things Infrastructure, Concepts and Challenges: an overview. Proceedings of the Fourth International Conference on Engineering \& MIS 2018. Anais...2018.

CHEN, J.-W. et al. A smartphone-based application for scale pest detection using multiple-object detection methods. Electronics, v. 10, n. 4, p. 372, 2021.

CRAIG, A. B. Understanding augmented reality: Concepts and applications. [s.1.] Newnes, 2013.

DA SILVA, D.; DA COSTA, C.; RIGHI, R. Um Modelo de Realidade Aumentada no âmbito do Turismo Ubíquo. Anais do VI Simpósio Brasileiro de Computação Ubíqua e Pervasiva. Anais...SBC, 2014.

DUBOIS, E.; NIGAY, L. Augmented Reality: Which Augmentation for Which Reality? Proceedings of DARE 2000 on Designing Augmented Reality Environments. Anais...: DARE '00.New York, NY, USA: ACM, 2000. Disponível em: <http://doi.acm.org/10.1145/354666.354695>. Acesso em: 16 dez. 2014

FERREIRA, F. H.; ARAÚJO, R. M.; SANTOS, R. P. DOS. Perspectivas para a Implantação de Câmpus Inteligentes. Anais da Escola Regional de Sistemas de Informação do Rio de Janeiro (ERSI-RJ). Anais... In: ANAIS DA V ESCOLA REGIONAL DE SISTEMAS DE INFORMAÇÃO DO RIO DE JANEIRO. SBC, 16 out. 2018. Disponível em: <https://sol.sbc.org.br/index.php/ersi-rj/article/view/4652>. Acesso em: 20 out. 2021

FERREIRA, F. H. C.; ARAÚJO, R. M. Campus Inteligentes: Conceitos, aplicações, tecnologias e desafios. RelaTe-DIA, 29 jan. 2018.

GEMAN, O. et al. Ubiquitous Healthcare System Based on the Sensors Network and Android Internet of Things Gateway. 2018 IEEE SmartWorld, Ubiquitous Intelligence Computing, Advanced Trusted Computing, Scalable Computing Communications, Cloud Big Data Computing, Internet of People and Smart City Innovation (SmartWorld/SCALCOM/UIC/ATC/CBDCom/IOP/SCI). Anais... In: 2018 IEEE SMARTWORLD, UBIQUITOUS INTELLIGENCE COMPUTING, ADVANCED TRUSTED COMPUTING, SCALABLE COMPUTING COMMUNICATIONS, CLOUD BIG DATA COMPUTING, INTERNET OF PEOPLE AND SMART CITY INNOVATION (SMARTWORLD/SCALCOM/UIC/ATC/CBDCOM/IOP/SCI). out. 2018.

GOOGLE MAPS. UFRB - Google Maps. Disponível em:

<https://goo.gl/maps/hF7qnQ1XCbtaMCh28>. Acesso em: 18 out. 2021.

GRUBERT, J.; GRASSET, R. Augmented reality for Android application development. [s.1.] Packt Publishing Ltd, 2013.

HASSAN, Z. S. Ubiquitous computing and android. 2008 Third International 
Conference on Digital Information Management. Anais... In: 2008 THIRD

INTERNATIONAL CONFERENCE ON DIGITAL INFORMATION

MANAGEMENT. nov. 2008.

HII, P.-C.; CHUNG, W.-Y. A Comprehensive Ubiquitous Healthcare Solution on an Android $^{\mathrm{TM}}$ Mobile Device. Sensors, v. 11, n. 7, p. 6799-6815, jul. 2011.

INSTAGRAM. Instagram. Disponível em: <https://instagram.com/>. Acesso em: 11 out. 2021.

IRWANSYAH, F. S. et al. Augmented reality (AR) technology on the android operating system in chemistry learning. IOP conference series: Materials science and engineering. Anais...IOP Publishing, 2018.

JAVORNIK, A. et al. Revealing the shopper experience of using a" magic mirror" augmented reality make-up application. Conference on designing interactive systems. Anais...Association for Computing Machinery (ACM), 2016.

JEONG, D. Road Damage Detection Using YOLO with Smartphone Images. 2020 IEEE International Conference on Big Data (Big Data). Anais...IEEE, 2020.

JIANG, Q. et al. Citizen Sensing for Improved Urban Environmental Monitoring. Research article. Disponível em:

<https://www.hindawi.com/journals/js/2016/5656245/>. Acesso em: $12 \mathrm{dez} .2017$.

KAJI, S. et al. Augmented reality in smart cities: applications and limitations. Journal of Engineering Technology, v. 6, n. 1, p. 18, 2018.

KAN, T.-W.; TENG, C.-H.; CHOU, W.-S. Applying QR code in augmented reality applications. Proceedings of the 8th International Conference on Virtual Reality Continuum and its Applications in Industry. Anais...2009.

KAVAKLI, M. A people-centric framework for mobile augmented reality systems (MARS) design: ArcHIVE 4Any. Human-centric Computing and Information Sciences, v. 5, n. 1, p. 37, 1 dez. 2015.

KO, S. M.; CHANG, W. S.; JI, Y. G. Usability principles for augmented reality applications in a smartphone environment. International Journal of HumanComputer Interaction, v. 29, n. 8, p. 501-515, 2013.

KUMAR, S. Challenges for Ubiquitous Computing. 2009 Fifth International Conference on Networking and Services. Anais... In: 2009 FIFTH INTERNATIONAL CONFERENCE ON NETWORKING AND SERVICES. abr. 2009.

LAWITZKI, P. Paul Lawitzki I software developer and game designer. Disponível em: <http://plaw.info/articles/sensorfusion/\#articles>. Acesso em: 20 out. 2021.

MADI, N. M.; ALBAKRY, N.; IBRAHIM, N. AR Mobile Application in Learning Hajj for Children in Malaysia: A Preliminary Study. 2020.

NABILA POPAL; RYAN REITH. Smartphone Market ShareSmartphone Market Share, 29 jul. 2021. Disponível em: <https://www.idc.com/promo/smartphone-market- 
share/os>. Acesso em: 23 set. 2020

PINTO, F. S.; CENTENO, J. A. S. A realidade aumentada em smartphones na exploração de informações estatísicas e cartográficas. Boletim de Ciências Geodésicas, v. 18, p. 282-301, 2012.

PIYARE, R. Internet of Things, Smart Home, Home Automation, Android Smartphone, Arduino. Internet of Things, p. 7, 2013.

Pokémon GO. Disponível em: <https://pokemongolive.com/>. Acesso em: 11 out. 2021.

POSLAD, S. Ubiquitous Computing: Smart Devices, Environments and Interactions. $1^{\text {a }}$ edição ed. Chichester, U.K: John Wiley \& Sons Inc, 2009.

RASHID, Z. et al. Using Augmented Reality and Internet of Things to improve accessibility of people with motor disabilities in the context of Smart Cities. Future Generation Computer Systems, v. 76, p. 248-261, 1 nov. 2017.

RAUSCHNABEL, P. A.; ROSSMANN, A.; TOM DIECK, M. C. An adoption framework for mobile augmented reality games: The case of Pokémon Go. Computers in Human Behavior, v. 76, p. 276-286, 2017.

REID, S. E. et al. Pervasive Mobile Services for Active Aging: An Exploratory Investigation into the Relationship Between Willingness-to-Adopt Mobile Devices and Shopping Experience. SpringerLink. Anais... In: INTERNATIONAL CONFERENCE ON SMART HOMES AND HEALTH TELEMATICS. Springer, Cham, 25 maio 2016. Disponível em: <https://link-springercom.ez67.periodicos.capes.gov.br/chapter/10.1007/978-3-319-39601-9_19>. Acesso em: 22 abr. 2017

RODELLO, I. A.; BREGA, J. R. F. Realidade virtual e aumentada em ações de marketing. Realidade Virtual e Aumentada: Aplicações e Tendências, v. 1, p. 44-57, 2011.

ROLIM, C. O. et al. An Ubiquitous Service-Oriented Architecture for Urban Sensing. SpringerLink, p. 1-10, 2015.

RUAN, K.; JEONG, H. An augmented reality system using Qr code as marker in android smartphone. 2012 Spring Congress on Engineering and Technology.

Anais...IEEE, 2012.

SHANMUGAPRIYA, M. Designing an M-Learning Application for a Ubiquitous Learning Environment in the Android Based Mobile Devices Using Web Services, 2011.

TAROUCO, L. M. R. et al. Internet das Coisas na Educação trajetória para um campus inteligente. Anais dos Workshops do Congresso Brasileiro de Informática na Educação, v. 6, n. 1, p. 1220, 27 out. 2017.

TENSORFLOW COMMUNITY. TensorFlow Lite Object Detection Android Demo. Disponível em: <https://github.com/tensorflow/examples>. Acesso em: 16 out. 2021. 
TZUTALIN. LabelImg. [s.l: s.n.].

WEISER, M. The Computer for the 21st Century. SIGMOBILE Mob. Comput. Commun. Rev., v. 3, n. 3, p. 3-11, jul. 1999.

WIBOWO, A.; HARTANTO, C. A.; WIRAWAN, P. W. Android skin cancer detection and classification based on MobileNet v2 model. International Journal of Advances in Intelligent Informatics, v. 6, n. 2, p. 135-148, 2020.

YOUNIS, A. et al. Real-time object detection using pre-trained deep learning models MobileNet-SSD. Proceedings of 2020 the 6th International Conference on Computing and Data Engineering. Anais...2020.

ZHOU, F.; DUH, H. B.-L.; BILLINGHURST, M. Trends in augmented reality tracking, interaction and display: A review of ten years of ISMAR. 2008 7th IEEE/ACM International Symposium on Mixed and Augmented Reality. Anais... In: 2008 7TH IEEE/ACM INTERNATIONAL SYMPOSIUM ON MIXED AND AUGMENTED REALITY. set. 2008. 